\title{
Design and Evaluation of an Adaptive Mobile Map-Based Visualisation System
}

\author{
Bradley van Tonder and Janet Wesson \\ Department of Computer Science and Information Systems, \\ Nelson Mandela Metropolitan University, PO Box 77000, Port Elizabeth, \\ South Africa, 6031 \\ \{Bradley.vanTonder, Janet.Wesson\} @nmmu.ac.za
}

\begin{abstract}
Mobile visualisation of map-based information is a difficult task. Designers of such systems must contend with the limitations of mobile devices in terms of hardware, screen size and input mechanisms. These problems are exacerbated by the nature of spatial data, where a large information space needs to be presented and manipulated on a small screen. In this paper, a prototype adaptive mobile map-based visualisation system, called MediaMaps, is presented. MediaMaps allows users to capture, location-tag, sort and browse multimedia in a map-based view. MediaMaps was designed to adapt the information visualised, the map-based visualisations and the supporting user interface. The results of an international field study, in which participants used MediaMaps on their personal mobile phones for a three-week period, are also presented. These results show that the adaptations implemented achieved high levels of accuracy and user satisfaction and successfully addressed some of the limitations of mobile map-based visualisation.
\end{abstract}

Keywords: Mobile map-based visualisation, adaptive interfaces, usability evaluation.

\section{Introduction}

Mobile devices have emerged in the last decade as a powerful new platform for the development of visualisation systems. These applications take advantage of two key features that distinguish mobile visualisation from desktop visualisation, namely the mobility of the platform and the ability to sense a wide range of variables influencing the user's context. Mobile visualisation systems, however, face a number of limitations in terms of resources, screen size and interaction mechanisms [1].

The problems associated with mobile visualisation are exacerbated in mobile map-based visualisation (MMV) systems, where a large information space must be visualised on a small screen. Several techniques have been developed to address these problems, including modified versions of desktop visualisation techniques and techniques which adapt various aspects of MMV systems.

Adaptive User Interfaces (AUIs) can provide potential benefits for addressing the limitations of mobile devices [2]. Adaptation of the user interface has been identified as an important aspect to be considered in the design of mobile visualisation systems 
[3]. Adaptation techniques include adaptation of the information visualised, the presentation of the map-based visualisations and the supporting user interface [4].

Usability evaluations and field studies of adaptive MMV systems are still rare. In this paper, the design and evaluation of an MMV system for capturing, locationtagging, organising and browsing multimedia collections is presented. This system, called MediaMaps, allows users to view map-based visualisations of their multimedia collections and implements information, visualisation and interface adaptation.

This paper is organised as follows. Section 2 presents related work concerning the problems associated with mobile visualisation and MMV and existing techniques for addressing these problems. Section 3 describes the design and implementation of MediaMaps, as well as the different forms of adaptation which were incorporated into the system. In Section 4, the results of a three-week international field study, in which participants used MediaMaps on their personal mobile phones, are presented.

\section{Related Work}

The problems and shortcomings associated with mobile visualisation and MMV are now described in more detail, followed by a brief discussion of existing techniques which have been developed to address these problems.

\subsection{Problems and Shortcomings}

Several problems and shortcomings related to mobile visualisation and MMV originate from the nature of mobile devices. The most obvious problem is the limited screen space available on mobile devices $[1,5]$. This is particularly problematic for MMV, in which a large information space needs to be visualised using the limited screen real estate available. Screen clutter can often result [6].

Another significant problem relates to the limited input mechanisms provided by mobile devices. These input mechanisms result in additional constraints on the interaction design of MMV systems [5, 7]. MMV systems typically allow the user to manipulate the map-based visualisations provided and support a wide range of fairly complex tasks [4]. The limited input mechanisms currently provided by mobile devices can be especially problematic in these systems. Mobile devices also provide limited processing power, memory and network bandwidth [1].

\subsection{Adaptive Mobile Map-Based Visualisation}

MMV systems can benefit significantly from personalisation techniques [8]. Several MMV systems have already been developed which incorporate adaptation. Existing research can be classified according to the variables which influence adaptation and the elements of the MMV system which are adapted.

Four classes of input variables have been identified in existing research $[4,8]$ :

- Device: The system can adapt to the differing capabilities of different devices.

- Context: The system can adapt to the user's context, including variables such as location, movement and ambient light. 
- Task: The system can adapt to the user's current activity. This could refer to a system task, or to an activity the user is conducting external to the system.

- User: The system can adapt to the user profile, or to the user's preferences, as determined by modelling the user's behaviour.

Adaptation has been incorporated into MMV systems in several ways. The following four broad classes of adaptation have been identified [4]:

- Information: The information selection and organisation can be adapted.

- Visualisation: The presentation of information can be adapted.

- User Interface: The supporting user interface can be adapted in a variety of ways, including the availability of functions and shortcuts.

- Device: Various aspects of the MMV system can adapt according to the device's capabilities.

Information adaptation has been implemented to adapt the amount, classification and level of detail of information [8, 9, 10]. Visualisation adaptation has been used to adapt the orientation, layout, section, scale, generalisation and graphical elements of map-based visualisations [4]. Adaptations of this type have ranged from simple adaptations of the map symbols used [11], to advanced techniques such as Focus Maps which uses generalization and colour to emphasise map regions considered relevant to the user's current task [12]. Interface adaptations are comparatively rare, but some have been implemented successfully in MMV systems [7]. Device adaptations are generally more low-level and ensure that the MMV system is able to run on different mobile devices $[9,10]$.

The next section describes the design and implementation of a prototype MMV system which includes information, visualisation and user interface adaptation.

\section{MediaMaps}

A prototype MMV system, called MediaMaps, was developed to incorporate the adaptation input variables and adaptation effects identified in Section 2.2. Device adaptation was excluded as it has been well-covered in existing research $[9,10]$ and is a technical issue which does not involve the user's behaviour or preferences.

\subsection{Functional Requirements}

MediaMaps provides the following basic functionality:

- $\quad$ Capturing and location tagging of multimedia (photos, videos and sound) (Figure 1).

- $\quad$ Organising multimedia into collections based on time and location (Figure 1).

- Map-based visualisation of multimedia collections and items (Figure 2).

- Search functionality allowing users to search by criteria such as date or location name (Figure 3).

Several systems have been developed to visualise photo collections on mobile devices. Many of these have incorporated context variables, including time and location, 
to allow users to browse their photo collections according to different dimensions [13, 14]. Zurfer, a mobile Flickr client, was also recently developed to allow users to browse both their own photos and those of their friends, organised into channels (based on spatial, topic or social dimensions) [15]. Existing mobile visualisation systems have, however, rarely made use of maps.

\subsection{Design and Implementation}

A model-based design approach was used to design MediaMaps, based on an existing model [16]. MediaMaps incorporates three components which facilitate adaptation, namely the User Monitoring and Modelling Component (UMMC), the Knowledge Base and the Adaptation Engine. MediaMaps was implemented using Java ME.

\section{User Monitoring and Modelling Component (UMMC)}

The UMMC monitors user interaction with the system and the values of context variables (the current time and the user's location). This information is processed using a user modelling component and stored in the User and Context Models. Privacy concerns are not an issue, as interaction data is stored locally on the user's personal mobile phone.

\section{Knowledge Base}

The Knowledge Base contains four different models which influence adaptation. These are the User and Context Models, as well as a Task Model and a System Model. The User Model stores user preferences relating to the different forms of adaptation implemented in MediaMaps. The Task Model is used to identify when the user is performing a new task (so that the system is able to adapt accordingly). The System Model is used to store the current values of the different adaptable parameters of the system. This acts as a central repository, ensuring that a consistent system state is maintained, as both the system and the user are able to perform changes to these parameters.

\section{Adaptation Engine}

The Adaptation Engine contains three components to manage each of the three forms of adaptation implemented in MediaMaps. The Information, Visualisation and Interface Manager components consult the Knowledge Base in order to ensure that the adaptations performed match the user's preferences, context and current task. These three forms of adaptation are discussed in more detail in the following sections.

\subsection{Information Adaptation}

Clutter is a significant problem in MMV systems [6]. The filtering of information according to user preferences was therefore considered to be a useful adaptation. This filtering is performed similarly to the visualisation adaptation in MediaMaps and is discussed in the next section. The limited interaction techniques currently available on mobile devices, combined with the increasing volumes of media items being stored, create a need for better ways of organising and retrieving multimedia on such devices. Furthermore, if a user's entire media collection is visualised in a single map-based 
view, screen clutter is likely to be severe. The sorting of media items into collections can help to minimise this clutter problem.

The real-time event detection (RED) algorithm, proposed by Chen and Chen [17], was used to implement information adaptation in MediaMaps. The RED algorithm allows photos to be sorted, creating collections based on time and location. The RED algorithm follows on a large amount of related work that attempts to organise photo collections based on a variety of variables, including time and location [18, 19, 20, 21]. Most existing algorithms are unsuitable in a mobile environment, as they are resource intensive and may result in significant delays before photos are sorted.

The RED algorithm provides for incremental sorting of photos in real-time (as they are captured). The RED algorithm was specifically designed for use in the resource-constrained environments of mobile phones. Photos can be classified as they are captured, allowing for immediate user feedback. This also means that the algorithm has the capability to adapt, with user responses to system recommendations used to improve the accuracy of the algorithm.

The RED algorithm organises the user's photo collection into "events", with the algorithm determining where the boundaries between these events lie. Chen and Chen hypothesised that, given certain assumptions, a particular user's photo capturing behaviour can be matched to a Poisson distributed statistical process.

The time gap between the current event and the previous event is used to decide which of the following three regions the current photo belongs to:

- $\quad R_{o}$ : Identified as belonging to the current (old) event.

- $\quad R_{l}$ : Falls into an intermediate area between a new or old event.

- $R_{n}$ : Identified as belonging to a new event.

If a newly captured photo falls into $R_{l}$, the distance between the location of the current photo and the preceding photo is used to determine whether a photo belongs to the current event or to a new event. The RED algorithm relies on three parameters that can be tuned according to user behaviour and feedback, $\mu_{d}$ (mean distance between photos), $\sigma_{d}$ (standard deviation) and $\lambda$ (arrival rate per hour). These three parameters are adjusted in order to compensate for errors made by the algorithm. For example, if a new photo is identified as belonging to $R_{o}$ (current event), but actually belongs to a new event, then the arrival rate, $\lambda$, needs to be increased to reflect the higher than expected rate of arrival of new events. For a detailed discussion of how the RED algorithm compensates for erroneous classifications, refer to [17].

The RED algorithm was used in MediaMaps to organise different multimedia types (including photo, video and sound recordings) into collections. Every time a user captures a media item, the system determines whether it belongs to the current collection, or to a new collection. The user is required to provide feedback regarding this recommendation (Figure 1). This feedback is used to update the algorithm's parameters. This feedback step can be removed once the system has learned the user's preferences. The version of MediaMaps used in the evaluation described in this paper required feedback for every media item captured.

The user's behaviour is monitored and recorded by the UMMC. The Context Model, which is updated with the user's position, is used to supply the location information for the algorithm. The parameters $\lambda, \sigma_{d}$ and $\mu_{d}$ are stored in the User Model and the Information Manager is used to perform changes to the underlying structure of the data. 


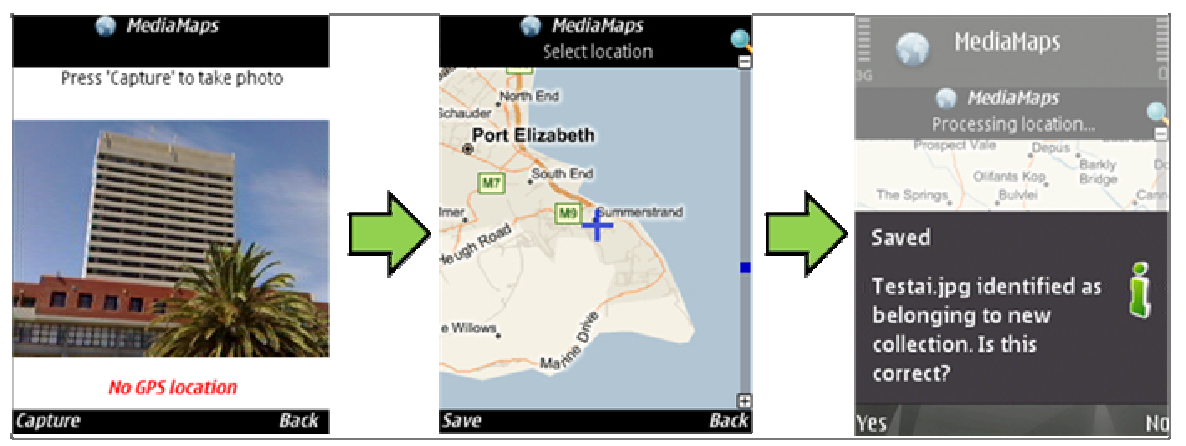

Fig. 1. Sorting media items into collections in MediaMaps. Manual location tagging is used in this case as the user's GPS location is unavailable.

\subsection{Visualisation Adaptation}

Several simple, yet important visual and information-related parameters were selected to be included in the adaptation process. The following parameters have previously been identified as important parameters to be adapted in MMV systems [4] and are adapted in MediaMaps:

- $\quad$ The zoom-level being visualised;

- The map-type being displayed;

- The latitude and longitude at which the map is centred;

- The time period being visualised; and

- The media types to be visualised.

One of the most significant problems of standard zooming and panning techniques is that the user often needs to perform many zooming and panning operations to find information of interest [22]. To overcome this, the map-based visualisations in MediaMaps are adapted according to previous user behaviour. This was done in order to save the user from having to perform the same view customisation operations each time a particular collection is viewed.

Naïve Bayesian classifiers were used to implement visualisation adaptation in MediaMaps. This technique assumes that different input variables are independent of one another. Despite the fact that this is typically not the case, this technique has proven accurate for several domains. Bayesian classifiers have previously been used in adaptive mobile applications [23] and were selected because of their simplicity and low resource requirements (while still providing potentially high levels of accuracy).

User behaviour is observed and recorded, with the current mode and the collection being viewed used as inputs in order to determine how to adjust these parameters. The UMMC observes and records user interaction information. This interaction data is then processed by the UMMC at system start-up in order to extract user preferences for different collections and visualisation modes. These preferences are recorded in the User Model. Older interaction data is progressively discarded to minimise the amount of interaction data that needs to be processed by the system. This also ensures that the adaptations keep pace with the user's changing preferences over time. 
The Visualisation and Information Managers are responsible for ensuring that the visualisation and information are adapted according to the user's preferences whenever a new visualisation is rendered. The Task Model is consulted to determine when a new visualisation is to be rendered in order to invoke these components.

Figure 2 shows the same data set (the icons indicate media collections) before and after adaptation. The map style, zoom level and location have all been adapted.

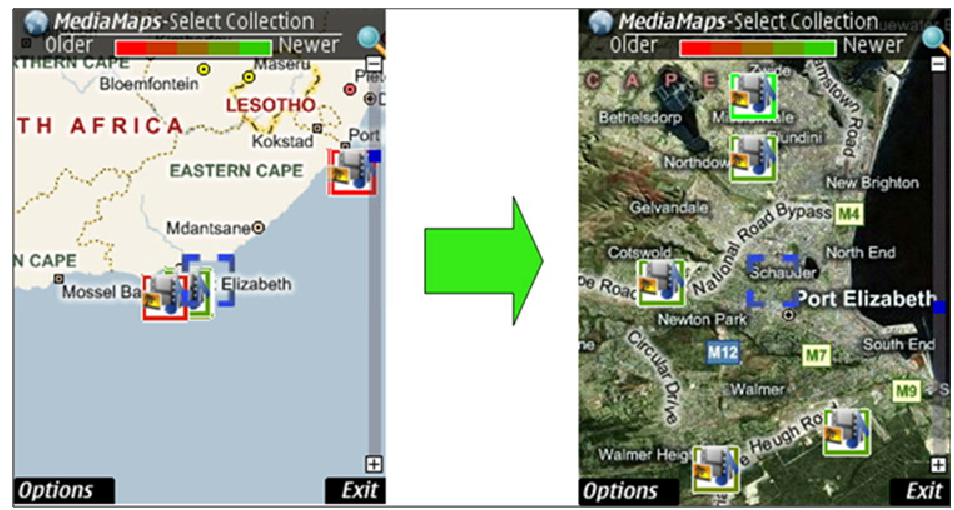

Fig. 2. Visualisation adaptation. The same data set is shown before and after adaptation.

\subsection{Interface Adaptation}

Interface adaptation in MediaMaps is limited to ordering of list options. Previous research has shown that the ordering of menus and lists in small screen devices can provide even greater efficiency benefits than in desktop systems [24]. Early user testing of MediaMaps showed that the users spent a significant amount of time browsing lists of options that can potentially grow quite large. As a result, it was decided to adapt the ordering of lists of options in MediaMaps based on how recently and frequently the different items are selected.

A variation of the algorithm used by Findlater and McGrenere [24] was used, which relies on the following three rules to order menu options:

1. The top section contains the most recently selected item and the two most frequently selected items

2. If there is duplication amongst these items, then the third most frequently selected item is included in the top section.

3. The items appear in the same relative order in the top section as they would in the bottom section of the list.

List items are moved to the top section, rather than replicated. Informal evaluation revealed that replicating items on short lists resulted in user confusion regarding which item to select. A grey background is used in order to draw users' attention to the fact that the top three items are adapted according to their behaviour (Figure 3). This interface adaptation is applied to all lists of options within the system, including the list of collections, list of saved views and lists of locations. Frequency data and the 


\begin{tabular}{|l|}
\multicolumn{2}{|c|}{ Select Suburb } \\
\hline Humewood \\
Walmer \\
Lorraine \\
Pari Park \\
Providentia \\
\hline Mount Pleasant \\
\hline Walmer Heights \\
Miramar \\
Lovemore Heights \\
Summerstrand \\
Charlo \\
Greenshields Park \\
Forest Hill \\
\hline Options \\
\hline
\end{tabular}

Fig. 3. Location search in MediaMaps, with recently and frequently selected items at the top

most recent selection for each list are stored in the User Model. The Task Model is used to determine when the user is accessing a list view and the Interface Manager is invoked in order to ensure that the appropriate options are in the top section of the list.

\section{Evaluation}

Preliminary user testing was conducted in order to evaluate the usability of the system and to ensure that no significant usability problems existed. The results of this evaluation revealed that MediaMaps was highly effective at supporting users in completing major system tasks. High levels of user satisfaction were also reported. Several minor usability problems were discovered and corrected. A longer term field study was then conducted to evaluate several aspects of the adaptations implemented. The results of this evaluation are discussed below.

\subsection{Objectives}

The field study was conducted to evaluate the following aspects of MediaMaps:

- Performance: Were the adaptations performed accurate?

- User satisfaction: Did users find MediaMaps easy to use? Were the adaptations performed perceived as useful?

These aspects were evaluated to determine whether the adaptations addressed the problems and shortcomings of MMV which were previously identified (Section 2.1).

\subsection{Participants}

International participants were recruited through a bidding process [25]. Twenty-six bids were accepted, although only 20 test participants (14 male, 6 female) completed the field study satisfactorily. Non-completion occurred for several reasons, including technical problems with the participants' phones and insufficient use of the system to 
warrant useful feedback. An average of $\$ 46.50$ was paid per test participant. Participants were recruited from twelve different countries on four continents. Figure 4 shows the demographic profile of the test participants.

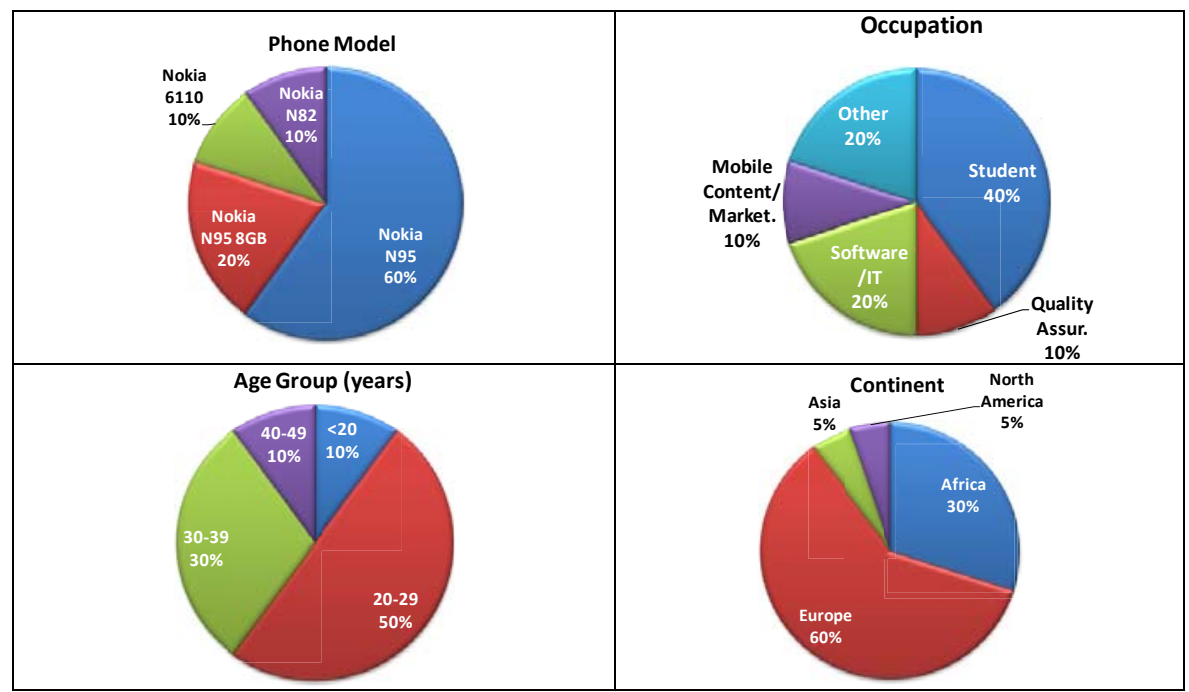

Fig. 4. Demographic profile of field study participants $(n=20)$

\subsection{Procedure}

Test participants were required to use MediaMaps on their personal mobile phones over a three week period. The participants were free to use MediaMaps as desired, but were encouraged to use the system as their primary means of capturing and browsing media for the duration of the test period. Participants were contacted in person, by email and instant messaging (where necessary) in order to ensure the system was being used as intended and to sort out any issues, particularly concerning installation.

At the end of the test period, users were required to complete a user satisfaction questionnaire based on the Questionnaire for User Interface Satisfaction (QUIS) [26]. A five point Likert scale was used with antonyms at extremes of each scale. A qualitative feedback section was also included to elicit positive and negative feedback, as well as suggestions for improvement. A logging mechanism was integrated into the system to record user and system behaviour.

\subsection{Performance Results}

Interaction $\log$ files were recorded to analyse the accuracy of the information and interface adaptations implemented in MediaMaps. Precision, Recall and F-Score metrics are commonly used to evaluate algorithms for detecting event boundaries in 
photo collections $[18,20]$. Precision refers to the ratio between the number of correctly detected event boundaries and the total number of event boundaries detected. Recall can be expressed as the ratio between correctly detected event boundaries and the actual number of event boundaries which exist. The F-score combines these two into a single metric $(F$-Score $=(2 x$ Precision $x$ Recall $) /($ Precision + Recall $))$. A general accuracy percentage was also calculated. A recommendation was regarded as accurate if it was accepted by the participant.

Table 1 shows the results obtained for Precision, Recall, F-Score and general accuracy. Cumulative results (across all participants) and average results (in which all participants received equal weighting) are shown. The average results for all four metrics were above $80 \%$. The accuracy of the algorithm was also analysed according to the three regions into which the RED algorithm classifies new media items (Section 3.3). It is interesting to note that the algorithm achieved high levels of accuracy $(85.55 \%)$ in $R_{l}$ where temporal information was inconclusive and location information was used to aid in the decision making process. Although this represents only seven percent of media items captured, it is nevertheless encouraging to note that the use of location information proved useful for ensuring accurate classifications.

The accuracy of the RED algorithm improved in response to user feedback. A significant correlation co-efficient of $0.79(\alpha=0.05)$ was calculated between the number of media items captured and the accuracy of the recommendations provided.

Table 1. Accuracy results for the RED algorithm implemented in MediaMaps

\begin{tabular}{lcc}
\hline Theme & Cumulative & Average \\
\hline Accuracy & $89.34 \%$ & $87.44 \%$ \\
Precision & $81.52 \%$ & $84.81 \%$ \\
Recall & $76.53 \%$ & $81.14 \%$ \\
F-Score & $78.95 \%$ & $83.33 \%$ \\
\hline
\end{tabular}

Accuracy information was also calculated for the interface adaptation algorithm. List option selections were split into three categories, namely pre-adaptive selections (when lists were too short to warrant adaptation), adaptive selections and nonadaptive selections. These results showed that (on average) options were selected from the adaptive section of the lists $76.78 \%$ of the time. Participant perceptions regarding the usefulness of the adaptation of list ordering were found to be positively correlated to the amount of time spent using MediaMaps. A statistically significant correlation co-efficient of $0.62(\alpha=0.05)$ was calculated.

\subsection{Satisfaction Results}

The results of the user satisfaction questionnaire are summarised in Table 2. All three forms of adaptation (Section D-F) were highly rated by participants (median=4.00). Participants were also highly satisfied with the support provided for searching for media items (Section C). 
Table 2. User satisfaction results summarised by section $(n=20)$

\begin{tabular}{lrrrc}
\hline Section & Mean & Median & Mode & Std. Dev. \\
\hline B. Capturing Media & 3.67 & 4.00 & 4.00 & 1.12 \\
C. Searching for Media & 3.90 & 4.00 & 5.00 & 1.11 \\
D. Sorting Media (information adaptation) & 3.83 & 4.00 & 4.00 & 1.02 \\
E. List Sorting (interface adaptation) & 3.72 & 4.00 & 5.00 & 1.25 \\
F. Map View Adaptation (visualisation adaptation) & 3.55 & 4.00 & 4.00 & 1.17 \\
\hline
\end{tabular}

The perceived usefulness of all three forms of adaptation in MediaMaps was highly rated by participants (where $1=$ Not useful and $5=$ Useful) (Table 3 ). Information adaptation, in which media items were sorted into collections based on time and location, was particularly highly rated. The perceived usefulness of all three forms of adaptation received median ratings of at least 4 . The results of Likert scale ratings also showed that participants found all three forms of adaptation to be logical.

Table 3. User satisfaction results for perceived usefulness $(n=20)$

\begin{tabular}{lrrrc}
\hline Adaptation Type & Mean & Median & Mode & Std. Dev. \\
\hline Information Adaptation (sorting of media items & 4.20 & 4.50 & 5.00 & 0.95 \\
into collections) & 3.80 & 4.00 & 5.00 & 1.24 \\
$\begin{array}{l}\text { Interface Adaptation (ordering of list options) } \\
\begin{array}{l}\text { Visualisation Adaptation (adaptation of map } \\
\text { visualisation parameters) }\end{array}\end{array}$ 3.75 & 4.00 & 4.00 & 1.02 \\
\hline
\end{tabular}

Qualitative feedback revealed that the system was used in a wide variety of situations and contexts. The field study took place during the northern hemisphere summer holidays and as a result, many of the participants used MediaMaps while on holiday and/or travelling between different cities and countries. General user satisfaction feedback was very positive regarding the usefulness, ease of use, learnability and enjoyment of MediaMaps.

Qualitative feedback regarding information adaptation supported the quantitative data, with many positive comments received. Sixty percent of participants specifically praised the information adaptation. The following positive comments regarding the information adaptation are examples of those received:

- " Excellent management capabilities for media items."

- "Very good system for organising your digital library."

- "Concept of collections and automated decisions very useful"

Participants also responded positively to the interface adaptation, commenting favourably on the "faster access to recent/frequent media collections". Visualisation adaptation was regarded as useful in the manual location-tagging map (Figure 1), with users not having to perform the same view customisation options repeatedly.

The only negative feedback received related to a lack of flexibility in the process of sorting media items into collections. Some participants wanted the ability to move 
items between collections after initial sorting, the ability to change the location associated with an image (in the case of incorrect manual tagging) and the ability to perform manual location-tagging at their own convenience. Some participants felt it unnecessary to create multiple collections for the same location on different dates. Many of these issues would be difficult to address without making significant changes to the RED algorithm.

Previous systems for browsing media collections on mobile devices have avoided the use of map-based interfaces $[14,15]$. It was therefore interesting to note that participants regarded the ability to view map-based visualisations of their media as highly useful (mean=4.25, median=4.50 and mode=5.00). Participants were also highly satisfied with the ease with which they were able to locate previously captured media using MediaMaps (mean=4.00, median=4.00 and mode=5.00).

\subsection{Analysis of Results}

The objective of the field study was to determine whether the information, visualisation and interface adaptations implemented in MediaMaps addressed some of the problems of MMV. Information adaptation was implemented in the form of an adaptive algorithm for sorting media items into collections. This was done to minimise user effort in organising media collections (using the limited interaction mechanisms available) and to reduce clutter that would otherwise result if a user's entire media collection was visualised in a single view. The high accuracy of the recommendations provided by the RED algorithm, combined with the positive participant feedback regarding the perceived usefulness of this form of adaptation, suggest that the information adaptation performed was indeed useful. Further supporting evidence regarding the usefulness of this form of adaptation is provided by the fact that participants were able to easily locate previously captured and sorted media items. The accuracy results achieved are particularly impressive if compared with those achieved by more resource-intensive algorithms, which do not allow media to be sorted in real time. Such algorithms have been shown to achieve only slightly superior accuracy, although tested on different media collections [18, 20]. The RED algorithm was also shown to adapt to user feedback and improve accuracy.

Chen and Chen evaluated the RED algorithm on a desktop computer. This research therefore represents the first time that the RED algorithm has been implemented and evaluated in a mobile context.

Visualisation adaptation was performed in order to reduce the amount of interaction required to perform view customisation operations such as zooming and panning. User satisfaction feedback showed that participants considered this form of adaptation to be very useful. Benefits were achieved in terms of efficiency and the amount of interaction required using the available interaction mechanisms.

Interface adaptation (list ordering) was implemented to improve user efficiency in selecting options from potentially long lists of options. Interaction logs showed that participants selected options from the adaptive portions of lists on average $76.78 \%$ of the time. This implies efficiency gains, as participants did not have to browse long lists searching for their desired option. Quantitative and qualitative user satisfaction feedback showed that the participants viewed this form of adaptation as highly useful, with perceived usefulness increasing the longer the participants used MediaMaps. 


\section{Conclusions}

This paper discussed the design and evaluation of an adaptive MMV system, called MediaMaps. MediaMaps provides automatic facilities for location tagging and organising media into collections on a mobile phone and allows users to view map-based visualisations of these collections. Existing adaptation techniques were modified for a mobile platform and extended to supported information, visualisation and interface adaptation. An international field study was conducted to evaluate the accuracy and perceived usefulness of the adaptation techniques and the participants' satisfaction with the system. The results of this study clearly show that the adaptation techniques were highly accurate and that the participants found the adaptations to be useful, efficient and easy to understand.

The evaluation of the RED algorithm used to perform information adaptation showed that this algorithm effectively supported the participants in organising and visualising their media collections. The accuracy of the RED algorithm was also shown to improve in response to user feedback. The problems inherent with the limited interaction mechanisms provided by mobile devices were minimised and effective use of the limited screen space was made by organising and visualising media collections based on time and location. The visualisation adaptation reduced the need for repetitive view customisation operations to be performed, while the interface adaptation minimised the amount of interaction necessary.

This paper thus provides empirical evidence that adaptation can provide significant benefits for mobile map-based visualisation. Future work will include changes to MediaMaps to address system limitations identified during the field study.

\section{References}

1. Chittaro, L.: Visualizing Information on Mobile Devices. IEEE Computer 39(3), 40-45 (2006)

2. Al-bar, A., Wakeman, I.: A Survey of Adaptive Applications in Mobile Computing. In: Proc. International Conference on Distributed Computing Systems (ICDCSW 2001), pp. 246-251. IEEE Computer Society Press, Los Alamitos (2001)

3. Karstens, B., Kreuseler, M., Schumann, H.: Visualization of Complex Structures on Mobile Handhelds. In: Proc. International Workshop on Mobile Computing (2003)

4. Reichenbacher, T.: Mobile Cartography - Adaptive Visualisation of Geographic Information on Mobile DevicesInstitut für Photogrammetrie und Kartographie, Technischen Universitat, Munchen (2004)

5. Björk, S., Redström, J., Ljungstrand, P., Holmquist, L.E.: PowerView: Using information links and information views to navigate and visualize information on small displays. In: Thomas, P., Gellersen, H.-W. (eds.) HUC 2000. LNCS, vol. 1927, pp. 46-62. Springer, Heidelberg (2000)

6. Baudisch, P., Rosenholtz, R., Halo, R.H.: A Technique for Visualizing Off-Screen Locations. In: Proc. SIGCHI Conference on Human Factors in Computing Systems (CHI 2003), pp. 481-488. ACM Press, New York (2003)

7. Carmo, M.B., Afonso, A.P., Pombinho de Matos, P., Vaz, A.: MoViSys - A visualization system for geo-referenced information on mobile devices. In: Sebillo, M., Vitiello, G., Schaefer, G. (eds.) VISUAL 2008. LNCS, vol. 5188, pp. 167-178. Springer, Heidelberg (2008)

8. Looije, R., te Brake, G., Neerincx, M.: Usability Engineering for Mobile Maps. In: Proc. International Conference on Mobile Technology, Applications, and Systems (Mobility 2007), pp. 532-539 (2007) 
9. Chalmers, D., Sloman, M., Dulay, N.: Map Adaptation for Users of Mobile Systems. In: Proc. World Wide Web Conference (WWW 2001), pp. 735-744. ACM Press, New York (2001)

10. Fresco, R., Marucci, L., Signore, O.: Adaptive Hypermedia for User-Centred Geovisualization in Multiplatform Environments. In: Proc. ACM Conference on Hypertext and Hypermedia (HyperText 2003), pp. 20-21. ACM Press, New York (2003)

11. Nivala, A.-M., Sarjakoski, L.: Adapting Map Symbols for Mobile Users. In: Proc. International Cartographic Conference, ICC 2005 (2005)

12. Zipf, A., Richter, K.-F.: Using Focus Maps to Ease Map Reading: Developing Smart Applications for Mobile Devices. Künstliche Intelligenz (KI) (Artificial Intelligence). Special Issue: Spatial Cognition (4), 35-37 (2002)

13. Harada, S., Naaman, M., Song, Y.J., Wang, Q., Paepcke, A.: Lost in Memories: Interacting With Photo Collections On PDAs. In: Proc. the Joint ACM/IEEE Conference on Digital Libraries (JCDL 2004), pp. 325-333. ACM Press, New York (2004)

14. Pauty, J., Couderc, P., Banâtre, M.: Using Context to Navigate Through a Photo Collection. In: Proc. International Conference on Human Computer Interaction with Mobile Devices \& Services (Mobile HCI 2005), pp. 145-152. ACM Press, New York (2005)

15. Naaman, M., Nair, R., Kaplun, V.: Photos on the Go: A Mobile Application Case Study. In: Proc. SIGHCI Conference on Human Factors in Computing Systems (CHI 2008), pp. 1739-1748. ACM Press, New York (2008)

16. van Tonder, B., Wesson, J.: Using Adaptive Interfaces to Improve Mobile Map-based Visualisation. In: Cilliers, C., Barnard, L., Botha, R. (eds.) Proc. SAICSIT 2008, pp. 257266. ACM Press, New York (2008)

17. Chen, W., Chen, M.: Event detection in real time on a personal camera phone image collection. Multimedia Systems (12), 479-492 (2007)

18. Naaman, M., Jiun Song, Y., Paepcke, A., Garcia-Molina, H.: Automatic Organization for Digital Photographs with Geographic Coordinates. In: Proc. CM/IEEE-CS Joint Conference on Digital Libraries (JCDL 2004), pp. 53-62. ACM Press, New York (2004)

19. Platt, J.C., Czerwinski, M., Field, B.A.: PhotoTOC: Automatic Clustering for Browsing Personal Photographs. In: Proc. the Fourth International Conference on Information, Communications and Signal Processing, pp. 6-10 (2003)

20. Cooper, M., Foote, J., Girgensohn, A., Wilcox, L.: Temporal Event Clustering for Digital Photo Collections. ACM Transactions on Multimedia Computing, Communications and Applications 1(3), 269-288 (2005)

21. Pigeau, A., Gelgon, M.: Building and Tracking Hierarchical Geographical \& Temporal partitions for Image Collection Management on Mobie Devices. In: Proc. the International Conference on Multimedia, pp. 141-150 (2005)

22. Kaptelinin, V.: A Comparison of Four Navigation Techniques in a 2D Browsing Task. In: Proc. SIGCHI Conference on Human Factors in Computing Systems (CHI 1995), pp. 282 283. ACM Press, New York (1995)

23. Boström, F., Floréen, P., Liu, T., Nurmi, P., Oikarinen, T.-K., Vetek, A., Boda, P.: Capricorn - An Intelligent User Interface for Mobile Widgets. In: Proc. the International Conference on Intelligent User Interfaces (IUI 2008), pp. 417-418. ACM Press, New York (2008)

24. Findlater, L., McGrenere, J.: Impact of Screen Size on Performance, Awareness and User Satisfaction With Adaptive Graphical User Interfaces. In: Proc. SIGCHI Conference on Human Factors in Computing Systems (CHI 2008), pp. 1247-1256. ACM Press, New York (2008)

25. Mob4Hire. Mob4Hire - Crowdsourced Mobile Application Testing, http : / / www . mob4hire. com

26. Chin, J.P., Diehl, V.A., Norman, K.L.: Development of an Instrument Measuring User Satisfaction of the Human Computer Interface. In: Proc. SIGCHI 1988, pp. 213-218. ACM Press, New York (1988) 